\title{
Blank trials and hypothesis behavior in young children*
}

\author{
KAREN G. FOREIT $\dagger$ \\ Brown University, Providence, R.I. 02912
}

The focusing behavior of second grade children was examined with four-dimensional problems under conditions of continuous feedback, continuous feedback with intertrial delay, and Levine's blank trials procedure. In the continuous outcome condition, only one blank trials probe was used per problem, inserted after varying numbers of successive outcome trials. The delay condition added a 15 -sec delay between outcome trials to the continuous outcome procedure. The standard blank trials procedure (probes following every outcome trial) produced significantly less focusing than continuous feedback; the delay condition fell in between and did not differ significantly from either.

The ability of a $\mathrm{S}$ to use a focusing strategy during concept identification-to eliminate hypotheses (Hs) after both positive and negative outcomes (Os) until only one logically correct $\mathrm{H}$ remains-is a function of both developmental level and the demands imposed by the task. Studies employing Levine's (1966) blank trials probe for $\mathrm{Hs}$ have shown that the performance of children improves with age (Eimas, 1969, 1970; Ingalls \& Dickerson, 1969) and with decreasing memory load (Eimas, 1970). The purpose of the present study was to investigate factors within the blank trials procedure itself which might elucidate this age-related deficit.

The use of blank trials probes, sets of trials for which no outcomes are given, increases the delay between outcome trials and adds noninformative trials (the blank trials themselves). To isolate and assess the effects of these two factors, three conditions were designed: blank trials (BL), continuous outcome (CONT), and continuous outcome-delay (DEL). The BL condition used Levine's standard procedure with probes throughout the problem. The CONT procedure used only one blank trials probe at the end of the problem, eliminating both increased intertrial delay and extra trials. The DEL condition added a 15-sec delay between outcome trials to the CONT procedure to increase intertrial delay without adding extra trials.

\section{METHOD}

\section{Subjects}

The Ss, 24 boys and 24 girls from the second grade of the Martin Luther King, Jr., School in Providence, R.I., were assigned randomly to the three experimental groups, with equal numbers of boys and girls in each group.

\section{Stimuli}

The experiment consisted of a series of eight practice problems and either 20 or 40 test problems. The problems

*The research reported was supported in part by funds from a Grant Foundation award to the Child Study Center of Brown University. This paper is sponsored by Peter D. Eimas, who takes editorial responsibility for it.

tNow at Brooklyn College of the City University of New York, Brooklyn, N.Y. 11210. consisted of four binary dimensions-size, color, letter, and position-presented simultaneously. Eight different colors and letters were combined in four different arrays such that each array contained different colors and letters from the other three; between successive problems, the color and letters were varied. Problem construction and use of blank trials followed the Levine (1966) procedure.

\section{Procedure}

Each S was tested individually with procedures similar to those used by Eimas (1969). Stimuli were presnted on a rear-projection screen. Ss responded by pointing; immediate verbal feedback was given by $\mathbf{E}$, followed by the next stimulus.

The practice problems familiarized $S$ with the blank trial stimuli and the permissible Hs. The test problems followed without introduction for the blank trials and continuous outcome groups. The BL condition followed Levine's procedure, 1 and used 20 test problems consisting of four repetitions of each of five predetermined outcome sequences $(---;+--;++-;-+-;+++)$. Test problems in the CONT condition consisted of one, two, or three successive outcome trials followed by a single blank trials probe. Thus, Hs could be probed at various stages of solution without adding either delay or extra trials. Forty problems, consisting of the five BL outcome sequences plus four partial-problem outcome sequences $(-;--;+-:++)$, were used to provide data comparable to those collected in the BL condition. The continuous outcome-delay condition used the CONT procedure with a 15 -sec delay between outcome trials prior to the blank trials probe. A filler task, counting forward or backward from a given number, was used to prevent rehearsal during the delay interval. These Ss practiced on the filler task before the test problems began. For all Ss, the trial following the last blank trials probe was reinforced randomly in half of the test problems and left blank in the other half.

\section{RESULTS AND DISCUSSION Blank Trials Data}

The mean percentages of $\mathrm{H}$-defining response sequences were $91 \%$ (DEL), 90\% (BL), 89\% (CONT). All were well above the chance level of $50 \%(\mathrm{p}<.01$ in all cases), and there were no significant differences between groups. Likewise, the mean percentages of outcome trial responses consistent with the blank trials responses immediately preceding were well above chance, with no significant differences between groups: $99 \%(\mathrm{BL}), 98 \%$ (DEL), and $97 \%(\mathrm{CONT})($ chance $=50 \% ; \mathrm{p}<.01$ in all cases).

\section{Focusing}

Measures of focusing evaluate S's ability to utilize and retain information. Two measures were derived for each $\mathrm{S}$ : percentage of focusing responses after each outcome trial (Hs consistent with feedback from all previous outcome trials), and from this percentage, the number of Hs in his $\mathrm{H}$ set (see Levine, 1966).

Only data following negative outcomes were included in these analyses. H-defining blank-trials responses and some verbal reports were used here. Whenever $S$ gave a random response pattern on the last blank trials set, at the end of the problem he was asked what his final $\mathrm{H}$ 
Table 1

Mean $\mathbf{H}$ Set Size as a Function of Treatments and the BlankTrials Sequence (Number of Preceding Outcome Trials)

\begin{tabular}{llll}
\hline & \multicolumn{3}{c}{ Blank-Trials Sequence } \\
\cline { 2 - 4 } Group & $\mathrm{H}_{1}$ & $\mathrm{H}_{2}$ & $\mathrm{H}_{3}$ \\
\hline Continuous & 5.03 & 3.58 & 4.10 \\
Delay & 4.55 & 4.57 & 5.11 \\
Blank Trials & 5.03 & 5.29 & 6.30 \\
\hline
\end{tabular}

had been. If his verbal report was consistent with his responses on three of the four blank trials and the following outcome trial. it was included.

As Table 1 illustrates, ${ }^{2}$ all groups showed an initial reduction in set size from the maximum of eight, but only the CONT group tended to eliminate Hs after the second outcome trial as well. A 2 (sex) by 3 (treatment) by 3 (trials) analysis of variance was performed on the percentages of focusing responses, normalized by the arcsin transformation. The treatment main effect missed significance, but the trials effect did reach significance $(\mathrm{F}>100, \mathrm{df}=2,84, \mathrm{p}<.001)$, as did the Treatment by Trials interaction $(\mathrm{F}=3.65, \mathrm{df}=4,84, \mathrm{p}<.01)$. After the second and third outcome trials, the blank trials group showed significantly less focusing than the continuous outcome group $(\mathrm{F}=6.40, \mathrm{df}=1,84, \mathrm{p}<.05$; $\mathrm{F}=4.00, \mathrm{df}=1,84, \mathrm{p}<.05)$, while the delay group fell midway between the other groups and did not differ significantly from either. The failure of either the BL-DEL or the DEL-CONT comparisons to reach significance suggests that the decrement produced by the blank trials probe was a function of both the delay and extra trials, since neither alone significantly reduced focusing.

\section{Coding, Recoding, and Retention}

The process of coding was assessed by the percentage of $\mathrm{Hs}$ consistent with a positive-outcome trial immediately preceding, and the process of recoding by the percentage of Hs consistent with an immediately preceding negative outcome. Retention effects were measured by comparing the percentages of $\mathrm{Hs}$ with outcome trials zero (C), one (C-1), and two (C-2) steps removed. Data entering into the $\mathrm{C}$ measure included all $\mathrm{Hs}$ and their immediately preceding outcomes. The $\mathrm{C}-1$ measure included $\mathrm{H}_{2}-\mathrm{O}_{1}$ (Hs manifest after the second $\mathrm{O}$ trial consistent with the first $\mathrm{O}$ trial) and $\mathrm{H}_{3}-\mathrm{O}_{2}$

Table 2

Mean Percentage of Hs Consistent With Positive $\left(\mathrm{O}^{+}\right)$or Negative (O-) Outcome Trials Immediately Preceding (C), One Step Removed (C-1), and Two Steps Removed (C-2) from the Measured $\mathrm{H}$

\begin{tabular}{|c|c|c|c|c|c|c|}
\hline \multirow[b]{2}{*}{ Group } & \multicolumn{2}{|c|}{$\mathrm{C}$} & \multicolumn{2}{|c|}{$C-1$} & \multicolumn{2}{|c|}{$\mathrm{C}-2$} \\
\hline & O+ & O- & $\mathrm{O}+$ & O- & $\mathrm{O}+$ & O- \\
\hline Continuous & 99 & 78 & 84 & 61 & 79 & 51 \\
\hline Delay & 98 & 80 & 79 & 54 & 69 & 51 \\
\hline Blank Trials & 98 & 77 & 77 & 52 & 70 & 54 \\
\hline
\end{tabular}

comparisons: and the C-2 measure included $\mathrm{H}_{3}-\mathrm{O}_{1}$ comparisons alone.

Table 2 presents the percentages of Hs consistent with positive and negative outcomes for each of the consistency measures. These data, normalized by the arcsin transformation, were entered into a 3 (treatment) by 2 (outcome) by 3 (distance) analysis of variance. The analysis showed dramatic effects of both outcome and distance ( $F>100, p<.001$, in both cases). Performance declined sharply over trials and between positive and negative outcomes, indicating that all Ss suffered from memory and recoding demands.

The Treatment by Outcome interaction failed to reach significance, suggesting that the experimental conditions operated nondifferentially on coding and recoding. The failure to find a significant Treatment by Distance interaction would also suggest a nondifferential effect on retention. However, this interaction need not be the only test for retention effects. Consistency measures confound retention and recoding effects whenever negative referent $\mathrm{O}$ trials contribute data to the analysis, as illustrated by the finding of a significant Outcome by Distance interaction $(\mathrm{F}=9.43$, df $=2,225$, $\mathrm{p}<.01)$ in the present study. Here $H$ consistency following negative $O s$ fell to chance after only one intervening trial, while $\mathrm{H}$ consistency following positive Os started higher and dropped more slowly. If retention effects are measured by $\mathrm{H}$ consistency following only positive referents, one recoding operation is eliminated and the confounding with recoding is reduced somewhat. Such a retention effect was found in the present data, in a significant Treatment by Outcome by Distance interaction $(F=3.00, \mathrm{df}=4,225, \mathrm{p}<.05)$. The locus of this interaction lies in the marginally significant advantage of the CONT group over the BL group following positive outcomes one and two steps removed from the referent $\mathrm{O}(\mathrm{t}=1.71, \mathrm{df}=30, \mathrm{p}<.10 ; \mathrm{t}=1.64$, $\mathrm{df}=30, \mathrm{p}<.10)$. No differential treatment effects following megative referent Os were found. This weak effect following positive trials suggests a possible retention problem in the present situation.

The present study clearly indicates that the use of blank trials probes reduces focusing in young children. It seems that the source of the debilitation lies at least in part in the retention processes, and that intertrial delay' and extra trials are both involved. Since young children are better focusers than their performance under a blank trials procedure would indicate, it may sometimes be advisable to adopt the continuous outcome procedure with a single probe.

\section{REFERENCES}

Eimas. P. D. A developmental study of hypothesis behavior and focusing. Journal of Experimental Child Psychology, 1969, 8, 160-172.

Eimas, P. D. Effects of memory aids on hypothesis behavior and focusing in young children and adults. Journal of Experimental Child Psychology, 1970, 10, 319-336.

Ingalls, R. P., \& Dickerson, D. J. Development of hypoisiesis behavior in human concept identification. Developmental Psychology, 1969, 1, 707-716. 
Levine, M. Hypothesis behavior in humans during discrimination learning. Journal of Experimental Psychology, 1966, 71, 331-338.

\section{NOTES}

1. A probe was inserted before the first outcome trial for reasons tangential to this paper: none of these probes were included in any analyses.

2. $\mathrm{H}_{2}$ refers to the $\mathrm{H}$ manifest immediately after the first outcome trial, $\mathrm{O}_{1} ; \mathrm{H}_{2}$ to the $\mathrm{H}$ after $\mathrm{O}_{2}$; and $\mathrm{H}_{3}$ to the $\mathrm{H}$ after $\mathrm{O}_{3}$.

(Received for publication July 30, 1973.)

\section{A comparison of $S+$ and $S$ - depression effects in differential conditioning*}

\author{
EARL R. McHEWITT \\ Bard College. Annandale-on-Hudson, N.Y. 12504
}

A total of 240 albino rats, across two experiments, received reward magnitude (differential or nondifferential) training in a nonchoice brightness discrimination apparatus. Results indicated significant $\mathrm{S}+$ and $\mathrm{S}$ - depression effects and showed that variability (among Ss) in performance to $\mathrm{S}$ - was greater than that to $\mathrm{S}+$ for the differentially rewarded Ss. Transformed speeds, taking into account the discrepant variances, indicated comparable $\mathrm{S}+$ and $\mathrm{S}$ - depression effects. The results were discussed in terms of current models of differential conditioning.

In differential conditioning, the finding that the performance of rats receiving differential reward is depressed to the small $(\mathrm{S}-)$ reward stimulus relative to that of a condition that receives small reward in both discriminanda is well documented and has received considerable theoretical attention (cf. Black, 1968; McHose, 1970). The similar observation for performance to the large (S+) reward stimulus, speeds being depressed relative to those of a group receiving large reward in both "S+" and "S-," has only recently received primary theoretical attention (cf. McHose, 1970: Perkins. 1970). There are several possibilities for this earlier neglect. First, early investigators. perhaps influenced by the perception literature, were interested in symmetrical contrast effects, depressed S- speeds and elated S+ speeds; thus, equal and depressed S+ speeds may have been viewed as a failure to demonstrate $\mathrm{S}+$ contrast (cf. Dunham, 1968). The graphical differences for these depression effects is perhaps yet another factor responsible for the emphasis on $\mathrm{S}-$ effects. S+ depression, based on mean speed differences. is much smaller than the similar comparison of $\mathrm{S}$ - speeds (Gavelek \& McHose, 1970: MacKinnon, 1967: Matsumoto, 1969). Also, the initial investigation in this

*This research was supported in part by Grant MH 10340 from United States Public Health Service to James H. McHose, Southern Illinois University, who sponsors the paper and takes full editorial responsibility for it. area did not obtain a statistically reliable $\mathrm{S}+$ depression effect (Bower, 1961). Finally, among the earlier approaches to these depression effects, one view is to attribute $\mathrm{S}$ - depression to the inhibitory properties of the small-reward stimulus. From this viewpoint, the inhibition is produced by discrepancies between the anticipated reward and that obtained in $\mathrm{S}-$, that is, the inhibition is frustration-based and develops, in primary form, in S- (cf. Bower, 1961; MacKinnon, 1967; Black, 1968). Not surprisingly, then, a frequent interpretation of the S+ depression effect is that it is in some sense a secondary phenomenon, resulting not from any inhibition generated in $\mathrm{S}+$, but from the generalization of inhibition which occurs in S- (e.g., Black, 1968; Henderson, 1966; Mackinnon, 1967); and, accordingly, the $\mathrm{S}$ - depression effect became the observation of primary theoretical interest.

As alternatives to the assumption that $\mathrm{S}+$ depression is a secondary phenomenon, more recent models provide theoretical precedent for treating $\mathrm{S}+$ depression as a phenomenon independent of S- depression (Perkins, 1970) and for the viewpoint that S+ and S- effects result from the same theoretical process (McHose, 1970: McHose, McHewitt, \& Peters, 1972). According to the latter framework, the extent of $\mathbf{S}+$ and $\mathbf{S}-$ depression is determined directly by the differences between the stimulus-specific incentives, and immediate implication being equal depression effects in performance to $\mathrm{S}+$ and S-.

Given these recent theoretical considerations, the reliability and the magnitude of $\mathrm{S}+$ depression should be firmly established. Theoretically, it would seem that. from one viewpoint, $\mathrm{S}+$ depression should be less than $\mathrm{S}-$ depression, and from another, $\mathrm{S}+$ depression should equal S-depression. As to the reliability of the effect. the most consistent finding is statistically reliable S+ depression (Gavelek \& McHose, 1970: MacKinnon. 1967; Matsumoto, 1969): yet, one of the most frequently cited studies (Bower, 1961) obtained only a graphical or directional S+ depression effect. The present study, combining data from two large $\mathrm{N}$ experiments. contributes further information as to the reliability and magnitude of the S+ depression effect. 